\title{
Sulfate Groups of the Mucilage of Red Seaweeds (Rhodopheceae)
}

\author{
Tooru Akahane and Sei-ichi IzUmi* \\ Department of Industrial Chemistry, Numazu Technical College, \\ Ooka, Numazu-shi, Japan \\ Received July 4, 1975
}

\begin{abstract}
It has been confirmed that there are two kinds of sulfate groups in red-algae mucilage which form thermal reversible gel, one of which is axial with respect to the pyranose ring and the other equatorial. The former forms 3,6-anhydro-L-galactose by desulfation and, at the same time, intermolecular interaction increases markedly due to hydrogen bonding, while in the latter this does not occur and, consequently, an increase in gelling ability is not observed.
\end{abstract}

It has been shown ${ }^{1 \sim 4)}$ that seaweeds with thermal reversible gel structure have the primary structure shown in Fig. 1. It is known that gelling ability of the agarose component, which contains a large amount of 3,6-anhydro-L-galactose $\left(\mathrm{G}_{3,6}\right)$, is much larger $^{5)}$ than that of the other components. The mechanism of gelation has been studied by Rees et al. ${ }^{6 \sim 9)}$ from the chemical point of view, based on investigation of the tertiary structure using X-ray analysis. McKinnon, et al. suggested there to be correlation between geration and a coil $\rightleftarrows$ helix transition by ORD experiments. On the other hand, Lawson et al. investigated ${ }^{11}$ from the biological point of view the change in structure of the native form by using transferase. All of these studies suggest intra- and intermolecular interaction in which $\mathrm{G}_{3,6}$ participates in gel formation and new intermolecular interactions. Desulfation by alkali treatment, widely used in industrial agatization of seaweeds for improving the gel strength, also forms $G_{3,6}$ during the process and this probably causes increases in mutual interaction mentioned above. However, $\mathrm{G}_{3,6}$ of all mucilages does not increase by the above-mentioned method as there is entirely no change after alkali treatment in mucilages of Funori (Gloiopeltis complanata). The authors investigated the correlation between the factors of gelation using $G$. complanata and Grasilaria verrucosa mucilages, and also the affect of the conformation of sulfate ester.

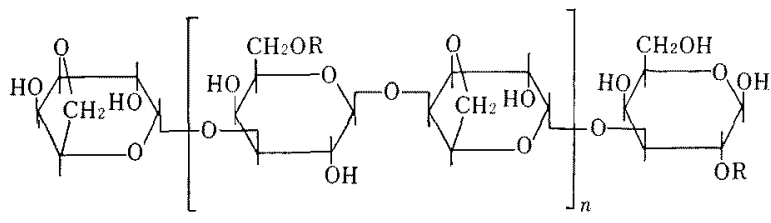

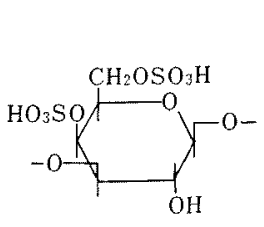

Sulfate groups

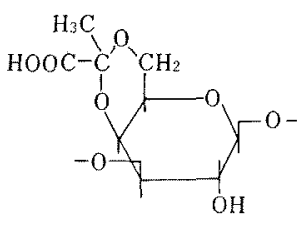

Pyruvic acid groups

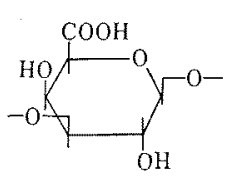

Uronic acid groups

FIG. 1. Primary Structure of Agar.

Upper main components (Agarose components)

Lower charged components (Agaropectin components)

* Present address: Nippon-kogyo, Co., Ltd., Takasago-cho, Mizushima, Japan. 


\section{EXPERIMENTAL}

\section{Extraction of mucilage}

Sample I, G. complanata (Itado, Shimoda-shi, Japan); II, G. verrucosa (Argentina and Chile); III, The above two samples desulfated.

Seaweeds I and II were washed with water, air-dried, extracted with hot water $\left(90^{\circ} \mathrm{C}\right)$ for $6 \mathrm{hr}$ and filtered through a bleached cotton cloth. Diatomaceous earth was added to the filtrate, which was filtered again through a glass filter. The crude mucilages were then reprecipitated with ethyl alcohol by the conventional method, centrifuged, and dried under reduced pressure below $50^{\circ} \mathrm{C}$.

Desulfation of the sample. The samples refined in this manner were dissolved in pure water and desulfated by treating with aqueous sodium hydroxide of various concentrations for $3 \mathrm{hr}$ at $50^{\circ} \mathrm{C}$.

Measurement of the number of acid groups and $G_{3,8}$ content. The number of acid groups was measured by electric conductivity titration. The concentration of hydrochloric acid used for making the sample for the acid types was $1.5 \mathrm{~N}$. The number of acid groups mole $/ \mathrm{C}_{\mathfrak{B}}$ was determined from the following relation: number of acid groups $=8.10 \times 10^{-3} \times V \times N / C$ where $V$, $C$, and $N$ represent the volume of potassium hydroxide, concentration of the sample solution and normality of potassium hydroxide, respectively. $\mathrm{G}_{3,6}$ content was obtained by colorimetric analysis after Yaphe et $a l^{0,103}$ using fructose as standard.

Conformational analysis of sulfate ester by infrared spectroscopy. Studies on the analysis of saccharides by IR have been concerned with conformational analy$\operatorname{sis}^{11 \sim 13)}$ of the various types of saccharide such as chondroitin sulfate $\mathrm{A}, \mathrm{B}$, and $\mathrm{C}$. The authors determined sulfate esters empilically on the basis of this work. Acetylated samples of $G$. complanata and $G$. verrucosa were used for comparative measurements. For acetylation, pyridine ${ }^{14}$ ) was used as catalyst and acetic anhydride as acetylating agent.

Determination of molecular weight and viscosity equation

Preparation of the fractionated sample. Fractionation of the sample was carried out by the precipitation method at room temperature using water (initial conc. of polymer: $5 \mathrm{wt} . \%$ as solvent and $95 \%$ ethyl alcohol as precipitant.

Measurement of number of various concentrations were prepared using a mixture of 4 mole/liter urea + $10^{-3}$ moles/liters sodium thiocyanate as solvent. Osmotic pressure $(\pi)$ was measured with an osmometer (type 503) made by Hewlett-Packard Co.

Number average molecular weight $(\overline{M n})$ was obtained by plotting $\pi / C$ against $C$ (wt. \% of sample solution), and extrapolating to find the value of $\pi / C(C \rightarrow 0)$ which equals $\overline{M n}$. The measurement temperature was $30 \pm$ $0.01^{\circ} \mathrm{C}$. The sample for osmometric experiment was prepared after repeating dialysis with solvent several 100 times, and dialysis equilibrium was determined by measuring the electric conductivity ${ }^{15}$ ) of the outside solution. Urea, a denaturant $t^{16,17}$ ) of proteines, was used in the present experiment as a gelation inhibiter.

Determination of the viscosity equation. First, the intrinsic viscosity $[\eta]$ of the various fractions was plotted against the values of $\overline{M n}$ on logarithmic graph paper under exactly the same conditions as used for measuring $\overline{M n}$, and viscosity constants ( $K$ and $\alpha$ ) were obtained from this graph.

\section{RESULT AND DISCUSSION}

\section{Conformation of sulfate groups in polysac- charides}

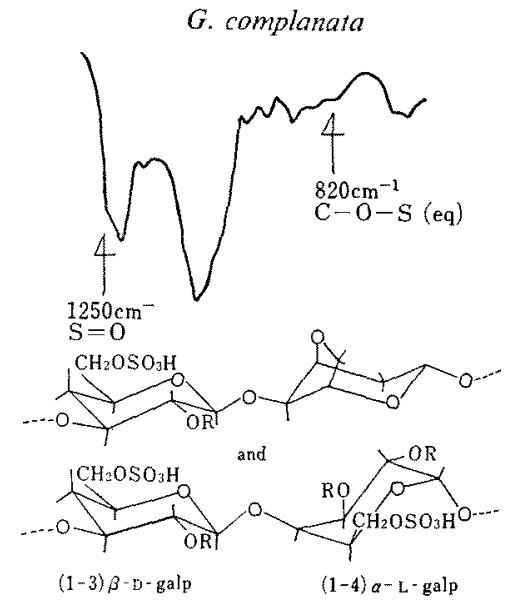

G. verrucosa
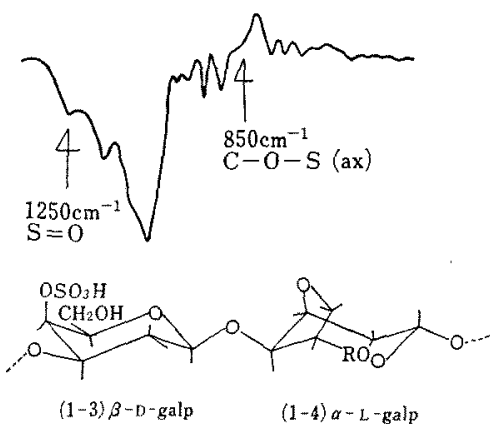

$\mathrm{R} ; \mathrm{H}$ or $\mathrm{SO}_{3} \mathrm{H}$

FIG. 2. Conformational Analysis of Mucilage by IR Spectroscopy. 


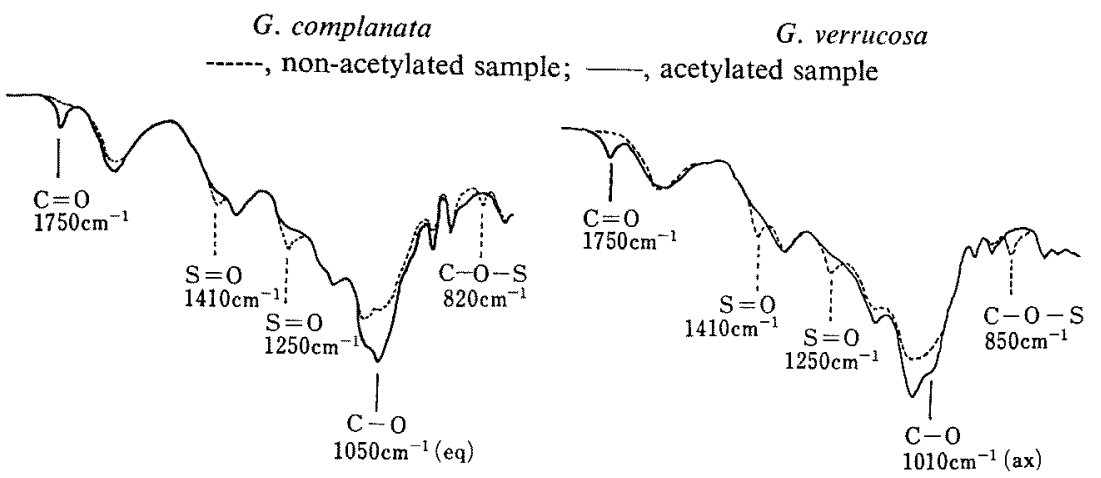

FIG. 3. IR Spectra of Acetylated and Non-acetylated Samples.

Absorption bands characteristic of sulfate groups disappeared and absorption bands at 1010 $\mathrm{cm}^{-1}$ or $1050 \mathrm{~cm}^{-1}$ due to acetyl groups appeared.

The results of IR analysis of the samples used are shown in Fig. 2. G. complanata and $G$. verrucosa were used as samples and the notable differences between them were as follows. Firstly, a large peak of $\mathrm{S}=\mathrm{O}$ which indicates the presence of sulfate groups was found at $1250 \mathrm{~cm}^{-1}$ with $G$. complanata, while the peak was small with $G$. verrucosa from Argentina and Chile. Secondly, C-O-S peak occured at $820 \mathrm{~cm}^{-1}$ in $G$. complanata, but at $850 \mathrm{~cm}^{-1}$ in $G$. verrucosa. The difference in sulfate groups stems from the physical properties of the mucilages and this is a large factor in the high solubility of the former in water. The difference in C-O-S absorption is due to the fact that the sulfate groups are boned equatorially (eq) with respect to the pyranose ring in $G$. complanata but are bonded axially (ax) in $G$. verrucosa, as shown below the IR spectra of each. In order to confirm this point, acetylated samples of both mucilages were prepared, the results of IR analysis being shown in Fig. 3. The absorptions at $1410 \mathrm{~cm}^{-1}, 1250 \mathrm{~cm}^{-1}$ and $820 \mathrm{~cm}^{-1}$ or 850 $\mathrm{cm}^{-1}$ disapeared in both acetylated mucilages, but absorptions near $1010 \mathrm{~cm}^{-1}$ and $1050 \mathrm{~cm}^{-1}$ due to streching vibration of $\mathrm{C}-\mathrm{O}$, believed to be that of acetyl groups, newly appeared, and according to the literature, ${ }^{18)}$ the corresponds to absorption of the axial group and the latter to the equatorial group, so that result which supports the interpretation of the $a b-$ sorption of C-O-S in Fig. 2 was obtained.
This difference in conformation has a deceptive effect on the process of desulfation and formation of $\mathrm{G}_{3,6}$.

Thus, sulfate esters have two kinds of conformation even in mucilages from the same red seaweeds and it was clear that the conformation of sulfate esters have a large influence on their physical properties, particularly $\mathrm{G}_{3,6}$ content. Factors (the number of acid groups, $\mathrm{G}_{3, \theta}$ content and molecular weight) affecting gelation of $G$. complanata and $G$. verrucosa which have a unique conformation of sulfate groups were studied.

\section{Correlation between desulfation treatment and} the number of acid groups and $\mathrm{G}_{3,6}$ content

Desulfation of $G$. complanata. As mentioned above, sulfate groups of polysaccharides can bond at any site $(\mathrm{OH})$ which are possible in galactose, but C-6 is structurally important in formation of $\mathrm{G}_{3,6} . \quad \mathrm{G}_{3,6}$ is formed by the following equation* by alkali treatment; an attempt was made to confirm whether $G$. complanata, in which the sulfate group at C-6 lie equatorially, forms $\mathrm{G}_{3,6}$ by desulfation. The results are shown in Table I.

The number of acid groups decreases with increasing sodium hydroxide concentration and it is clear that desulfation proceeds. It

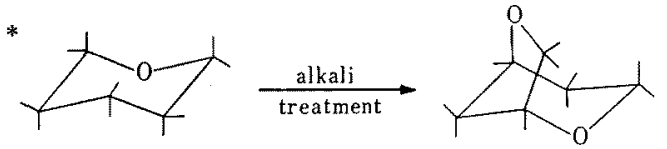


Table I. The Relation between NaOH Concentration, Density of Acid Groups AND 3,6-ANHYDRO-L-GARACTOSE

Reaction time: $3 \mathrm{hr}$. Temperature: $50^{\circ} \mathrm{C}$. Sample conc.: 1 wt. $\%$. Sample: G. complanata.

\begin{tabular}{|c|c|c|c|c|}
\hline $\begin{array}{c}\mathrm{NaOH} \\
(\%)\end{array}$ & $\begin{array}{c}\mathrm{OSO}_{3} \mathrm{H} \\
\left(\mathrm{mole} / \mathrm{C}_{8}\right)\end{array}$ & $\begin{array}{c}\mathrm{COOH} \\
\left(\mathrm{mole}_{\mathrm{C}} \mathrm{C}_{6}\right)\end{array}$ & $\begin{array}{c}\text { Total } \\
\text { acid } \\
\left(\text { mole } / C_{\theta}\right)\end{array}$ & $\begin{array}{c}\text { 3,6-Anhydro- } \\
\text { L-galactose } \\
(\%)\end{array}$ \\
\hline 0 & 0.032 & 0.019 & 0.052 & 25.1 \\
\hline 0.03 & 0.023 & 0.013 & 0.036 & 23.4 \\
\hline 0.10 & a) & 0.011 & 0.011 & 21.6 \\
\hline 0.50 & a) & 0.007 & 0.007 & 18.1 \\
\hline 1.50 & a) & 0.005 & 0.005 & 16.1 \\
\hline
\end{tabular}

a) Desulfation.

was considered previously that the alkali treatment acts only on sulfate groups and that carboxyl groups are not affected. However, carboxyl groups also decreased gradually in the present experiment. This is thought due to the fact that cleavage of the main chain takes place and the residue dissociates during refining due to centrifugal separation because the sample of this type is a polysaccharide of relatively low molecular weight. The same can also be stated with respect to the tendency of $\mathrm{G}_{3,6}$ content to decrease, but, what is more important than this with regard to $G_{3, e}$, is that despite the expectation that there would be no increase in $\mathrm{G}_{3, s}$ content even when desulfation proceeded, $\mathrm{G}_{3,6}$ content in fact decreased for the same reason as in the case of carboxyl groups. This fact was confirmed by the previous IR information. It was found that $\mathrm{G}_{3,6}$ is not formed when the sulfate groups at C-6 lies equatorially and $\mathrm{G}_{3,6}$ dissociates by depolymerization similar to the case of carboxyl groups.

Desulfation of $G$. verrucosa. For comparison with $G$. complanata, the relation between desulfation and $\mathrm{G}_{3,6}$ formation in two kinds of $G$. verrucosa, which have been made into commercial products by alkali treatment, is shown in Table II. These differ from $G$. complanata as the content of $\mathrm{G}_{3,6}$ increases with the progress of desulfation. This tendency can be seen in almost all sulfate groups with the exception of Funori such as $G$. complanata and it indicates the progress of
Table II. The Relation between NaOH CONCENTRATION AND 3,6-ANHYDRO-L-Galactose

Sample: $G$. verrucosa (Chile and Argentina). Alkali treatment: various conc. of $\mathrm{NaOH}, 60^{\circ} \mathrm{C}, 3 \mathrm{hr}$.

\begin{tabular}{rccccc}
\hline \multirow{2}{*}{$\begin{array}{c}\mathrm{NaOH} \\
(\%)\end{array}$} & \multicolumn{2}{c}{$\begin{array}{c}\text { Density of sulfate } \\
\text { esters }\end{array}$} & \multicolumn{2}{c}{$\begin{array}{c}\text { 3,6-Anhydro-L- } \\
\left.\text { (mole/ } \mathrm{C}_{6}\right)\end{array}$} & \multicolumn{2}{c}{ galactose } & $(\%)$ \\
\cline { 5 - 6 } & Chile & Argentina & Chile & Argentina \\
\hline 0 & 0.045 & 0.06 & 26.8 & 33.7 \\
4 & 0.033 & 0.06 & 28.8 & 34.4 \\
7 & 0.030 & 0.04 & 33.9 & 31.4 \\
10 & 0.027 & 0.01 & 38.0 & 31.4 \\
\hline
\end{tabular}

alkali treatment $\rightarrow$ desulfation $\rightarrow$ formation of $\mathrm{G}_{3,6}$ and increase in gelling ability. In other words, it explains the fact that sulfate groups are presents at C- 6 . Also, the $\mathrm{G}_{3,6}$ formation of the same verrucosa mucilage differs but it is assumed that this can reasonably be explained as follows.

Generally, $\mathrm{OH}$ at C-2, C-4 and C-6 can bond sulfate groups of acid polysaccharides. Firstly, the sulfate group of $G$. verrucosa (Chile) is considered to be at C-6, because $\mathrm{G}_{3,6}$ increases with progress of alkali treatment. Next, $G$. verrucosa (Argentina) is considered to contain a large amount of $G_{3,6}$ prior to alkali treatment because sulfate groups attached to positions (C-2 or C-4) other than the C-6 are desulfated.

Determination of molecular weight and viscosity equation. The sample is fractionated $G$. complanata by precipitants (ethyl alcohol of various conc.).

$\overrightarrow{M n}$ of the polymer fraction to $[\eta]$ under the conditions shown in Fig. 4. Values of $\alpha=$ 1.23 and $K=1.92 \times 10^{-4}$ were obtained from the gradient of this straight line. The viscosity equation was therefore $[\eta]=1.92 \times$ $10^{-4} \bar{M}_{v}^{1,23}$, where $\bar{M}_{v}$ represent the viscosity average molecular weight. Also, the value of $\alpha$ suggests that the molecules of this sample are rod-shaped and have little flexibility.

Also, $\overline{M n}$ is very small compared with that of similar polysaccharides and it is clear from this also that the gelling ability of this mucilage is low.

Correlation between molecular weight, the number of acid groups and $G_{3,6}$ content. Mole- 


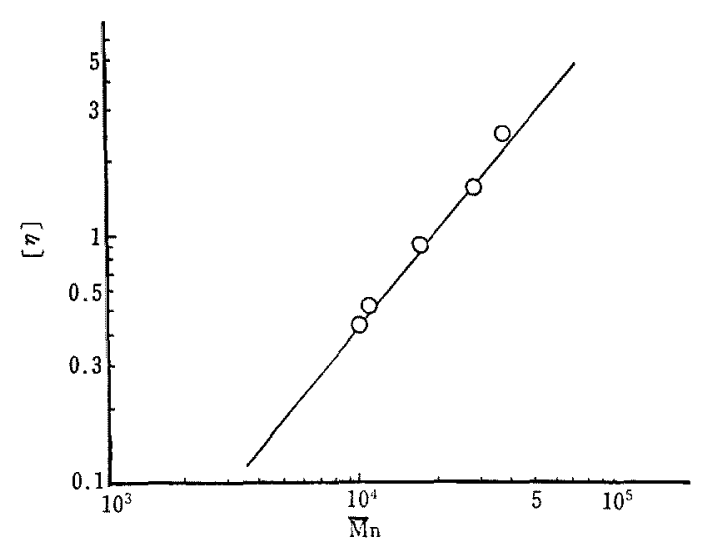

FIG. 4. Plots of Staudinger Index [ $\eta]$ against Molecular Weight $(\overline{M n})$ by Osmometry.

$\bar{M} v$ : viscosity average molecular weight.

Temperature: $30^{\circ} \mathrm{C} \pm 0.01^{\circ} \mathrm{C}$. Solvent: 4 mole/liter $+10^{-3}$ sodium thiocyanate. Sample: $G$. complanata.

$$
\begin{aligned}
a & =1.23 \\
K & =1.92 \times 10^{-4} \\
{[\eta] } & =1.92 \times 10^{-4} \bar{M}_{v}^{1.23}
\end{aligned}
$$

cular weight, the number of acid groups and $\mathrm{G}_{3,6}$ content of the same fraction of $G$. complanata are shown Fig. 3.

Firstly, it can be seen that the number of acid groups has random distribution without relation to $\overline{M n}$. Next, there is a tendency for higher $G_{3,6}$ content with lower molecular weight. It is not clear whether this result is specific to $G$. complanata.

Table III. The Relation between Moleculr Weight, Density of ACID Groups, AND 3,6-ANHYDRO-L-GALACTOSE

Sample: $G$. complanata.

\begin{tabular}{rcccc}
$\overline{M n} \times 10^{-3}$ & $\mathrm{OSO}_{3} \mathrm{H}$ & $\mathrm{COOH}$ & $\begin{array}{c}\text { Total } \\
\text { acid } \\
\left(\mathrm{mole} / \mathrm{C}_{8}\right)\end{array}$ & $\begin{array}{c}\text { 3,6-Anhydro } \\
\left(\mathrm{mole} / \mathrm{C}_{6}\right)\end{array}$ \\
$\begin{array}{rcccc}\text {-L-galactose } \\
(\%)\end{array}$ \\
\hline 76 & 0.176 & 0.111 & 0.287 & 1.4 \\
52 & 0.271 & 0.097 & 0.368 & 15.4 \\
38 & 0.032 & 0.019 & 0.052 & 18.5 \\
14 & 0.168 & 0.105 & 0.272 & 23.2 \\
4 & 0.325 & 0.181 & 0.506 & 27.6 \\
\hline
\end{tabular}

Previously, factors (the number of acid groups, molecular weight and $\mathrm{G}_{3,6}$ content) have been considered to affect the gelation of mucilages, but it has been found in the present experiments that the conformation of sulfate groups, that is, whether sulfate groups are present at C-6, is also an important factor.

Acknowledgement. The authors wish to express their apprecation to Plof. H. Noda of Tokyo University for the osmotic pressure measurements.

\section{REFERENCES}

1) C. Araki, Bull. Chem. Soc. Japan, 29, 543 (1956).

2) C. Neuberg and H. Ohle, Biochem. Z., 125, 311 (1921).

3) C. Araki, Nippon Kagaku Kaishi, 58, 1213 (1937).

4) S. Hirase, Bull. Chem. Soc. Japan, 30, 68 (1957).

5) K. Katsuura, T. Fuse and K. Kano, Kogyo Kagaku Zasshi, 68, 205 (1965).

6) D. A. Rees and J. W. B. Samuel, J. Mol. Biol., 48, 85 (1969).

7) A.A. McKinnon, D.A. Rees and F.B. Williamson, Chem. Commun., 1969, 701.

8) C. J. Lawson and D. A. Rees, Nature, 227, 392 (1970).

9) W. Yaphe, Anal. Chem., 32, 1327 (1960).

10) W. Yaphe and G. P. Arsenault, Anal. Biochem., 13, 145 (1965).

11) S. F. D. Orr, Biochim. Biophys. Acta, 14, 173 (1954).

12) K. Onodera, S. Hirano, N. Kashimura and F. Masuda, presented at the International Symposium on the Chemistry of Natural Products, Kyoto, Japan, Apr. 12 18, 1964.

13) H. Spending, Advan. Carbohydr. Chem., 19, 23 (1964).

14) M. Nagasawa, A. Takahashi, M. Izumi and $I$. Kagawa, J. Polym. Sci, 38, 213 (1959).

15) S. A. Pice and P. Doty, J. Am. Chem. Soc., 79, 3937 (1957).

16) K. Katsura, T. Akahane and S. Abe, Nippon Kagaku Zasshi, 89, 983 (1968).

17) C. H. Hadson and J. K. Dale, J. Chem. Soc., 37, 1264 (1915).

18) Y. Nitta, Y. Nakajima, M. Kuranari, A. Momose and J. Ide, Yakugaku Zasshi, 81, 1160 (1960). 